children with acquired oesophageal strictures, the possibility of a chronic impacted foreign body should be kept in mind.

\section{Final diagnosis}

Stricture of the oesophagus by a mediastinal mass caused by a chronically impacted radiolucent foreign body.

Keywords: oesophageal strictures; foreign body ingestion

1 Myers NA. Esophageal disorders associated with emesis in Myers NA. Esophageal disorders associated with emesis 2 infants and children. Semin Pediatr Surg 1995;4:166-75. and dysphagia because of prolonged esophageal foreign and dysphagia because of prolonge

3 Remsen K, Biller HF, Lawson W, Som ML. Unusual presentation of penetrating foreign bodies of the upper aerodigestive tract. Ann Otol Rhinol Laryngol 1983;92:3244

\section{Learning points}

In children with acquired oesophageal strictures

- even without a definite history of ingestion, there could be a foreign body in the oesophagus

- diagnostic work-up in cases of suspected 'occult foreign body' still presents difficulties, particularly if the foreign body is radiolucent

- the chronic presence of a foreign body in the oesophagus may lead to severe and unsuspected complications a long time after ingestion

Box 2

4 Herman TE, McAlister WH. Esophageal diverticula in childhood associated with strictures from unsuspected foreign bodies in the esophagus. Pediatr Radiol 1991;21: $410-2$.

5 Fernandes ET, Hollabough RS, Boulden T. Mediastina mass and radiolucent esophageal foreign body. $\mathcal{F}$ Pediatr Surg 1989;24:1135-6.

\title{
Increasing need for replacement therapy in long-standing Addison's disease
}

\author{
L Ranganath, S R Gould
}

A diagnosis of Addison's disease was made in a woman at the age of 23 years. She was commenced on replacement therapy with cortisone acetate $25 \mathrm{mg}$ and fludrocortisone $50 \mu \mathrm{g}$ in the morning and cortisone acetate $25 \mathrm{mg}$ in the evening; her adrenal insufficiency was well controlled on this replacement therapy. Since the age of 46 years increasing doses of mineralocorticoid and glucocorticoid were administered to control her symptoms. At the age of 52 years she was taking hydrocortisone $40 \mathrm{mg}$ and fludrocortisone $200 \mu \mathrm{g}$ in the morning and hydrocortisone $20 \mathrm{mg}$ in the evening. Despite this increase in dose of glucocorticoid and mineralocorticoid she felt tired and unwell. She was a keen horsewoman and had always been underweight. She had become menopausal at the age of 28 years and had been put on oestrogen replacement therapy with Prempak C $(0.625 \mathrm{mg})$. Despite the increasing dose of hydrocortisone she remained thin (weight

Epsom General

Hospital, Epsom, KT18

7EG, UK

Department of

Chemical Pathology

L Ranganath

Department of

Medicine

S R Gould

Accepted 19 November 1997 $48.6 \mathrm{~kg}$, Body Mass Index $17.8 \mathrm{~kg} / \mathrm{m}^{2}$ ). Her blood pressure was $130 / 80 \mathrm{mmHg}$ in the recumbent posture and there was no postural drop.

\section{Questions}

1 Why is the dose of replacement steroids increasing?

2 What further investigations would you perform?

3 What is the diagnosis in this woman?

4 What tests would you perform in following this patient up? 
Answers

QUESTION 1

The absence of signs of steroid excess in the context of increasing doses of gluco- and mineralo-corticoid therapy is suggestive either of malabsorption or increased catabolism.

\section{QUESTION 2}

Measurement of serum cortisol following oral administration will either confirm or refute malabsorption of steroids. An important cause of increased catabolism is hyperthyroidism, which is especially likely in the context of autoimmune endocrinopathy in this patient. Thyroid function tests should be performed.

\section{QUESTION 3}

This woman has Addison's disease, premature menopause and possible hyperthyroidism and is likely to have type II auto-immune polyendocrinopathy (which also includes insulindependent diabetes mellitus (IDDM), vitiligo, alopecia, pernicious anaemia, coeliac disease and myasthenia in various combinations). Auto-immune polyendocrinopathy is now classified as types I, II and III. ${ }^{12}$ Type I, inherited as an autosomal recessive trait, usually presents in the first decade of life as a triad of mucocutaneous candidiasis, hypoparathyroidism and Addison's disease. Type III, inherited as an autosomal dominant trait like type II, is a combination of IDDM, Addison's disease and Hashimoto's hypothyroidism.

\section{QUESTION 4}

The other features of polyendocrinopathy should be anticipated and screened prospectively every 2-4 years. This would involve appropriate history taking, fasting plasma glucose, full blood counts, serum B12 and autoimmune profile including antigliaden and gastric antiparietal cell antibodies. Appropriateness of therapy for her polyendocrinopathy should be monitored by serological tests and clinical response. This endocrinopathy can be inherited as an autosomal dominant trait and family members should be screened. Coeliac disease should be excluded in view of the low body weight in this patient by antigliaden antibodies and jejunal biopsy following endoscopy. Although this patient is on oestrogen replacement therapy, a bone density scan would be useful to allow early additional therapy for established osteoporosis using agents such as bisphosphonates.

\section{Clinical course}

Her pulse rate was 84 beats/min and regular. There was no thyroid enlargement and there were no eye signs consistent with thyrotoxicosis. It was unclear whether the increasing requirement for hydrocortisone and fludrocortisone was due to decreased absorption from the intestinal lumen or to increased catabolism post-absorption. Blood samples were taken both before and after her morning dose of hydrocortisone for cortisol measurement (figure). The cortisol peak was satisfactory,

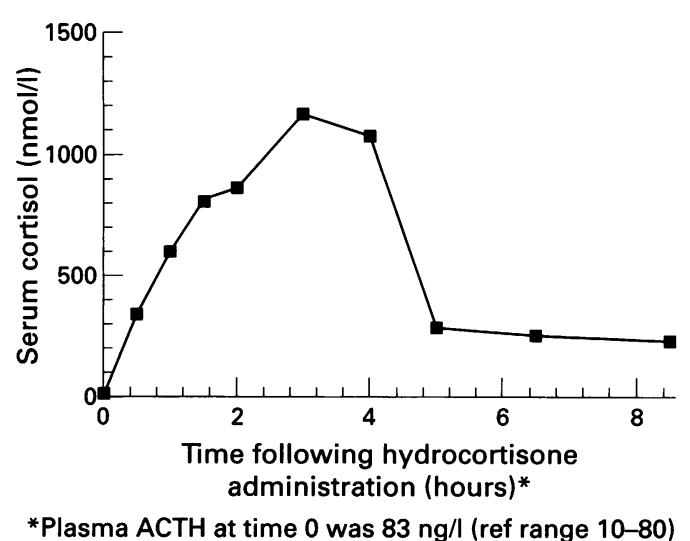

Figure Profile of serum cortisol following oral administration of $40 \mathrm{mg}$ hydrocortisone

confirming adequate gastrointestinal absorption. Serum electrolytes, urea, creatinine, calcium, liver function tests and glucose were normal. Haemoglobin, full blood count and erythrocyte sedimentation rate were also normal. Thyroid function tests revealed a total thyroxine $218 \mathrm{nmol} / 1$ (normal 50-160), free tri-iodothyronine $13 \mathrm{pmol} / \mathrm{l}$ (normal 3-9), and serum thyrotropin less than $0.2 \mathrm{mU} / 1$ (normal 0.5-5.5), confirming subclinical hyperthyroidism. She became euthyroid following propylthiouracil $200 \mathrm{mg}$ daily and the dose of hydrocortisone was reduced to previous levels. She became hypokalaemic and the dose of fludrocortisone was reduced. Family screening, serum B12, endoscopy with jejunal biopsy and bone density scan were arranged.

\section{Discussion}

The patient described in this report was on replacement therapy for Addison's disease which had led to stable control for more than two decades. The increased dose requirements for hydrocortisone and fludrocortisone prior to the recent investigations did not lead to weight gain or iatrogenic Cushing's syndrome. The question, therefore, was whether she was absorbing cortisol adequately or whether there was an acceleration in steroid breakdown. The cortisol day profile showed a good intestinal absorption of orally administered cortisol. This suggested that our patient had increased catabolism of cortisol. In view of her adrenal and gonadal failure we pursued the possibility of an additional endocrine abnormality to account for the increased catabolism of hydrocortisone.

Thyrotoxicosis is associated with increased breakdown of gluco- and mineralo-corticoids as well as oestradiol. ${ }^{3}{ }^{4}$ In the absence of adrenal or gonadal failure, this increased breakdown is accompanied by compensatory increases in synthesis and secretion of these hormones due to the presence of intact feedback regulatory loops. When failure of adrenal and gonadal function occur due to end-organ failure, increased catabolism of exogenously administered gluco- and mineralo-corticoids, as well as oestradiol therapy, cannot be compensated for by an 
increase in endogenous synthesis and secretion. This may lead to re-appearance of symptoms of deficiency leading to increasing requirements for the replaced hormones. Even mild or early hyperthyroidism may result in increased dose requirements and lead to its early recognition. Our patient did not show re-appearance of menopausal symptoms; we wonder whether the conjugated equine oestrogens she was taking were catabolised more slowly than natural oestradiol, offering greater oestrogenisation.

Hyperthyroidism may have been present undetected in our patient for some time. She had several risk factors for osteoporosis such as a thin body frame, early menopause, undetected hyperthyroidism, probable increased catabolism of oestrogen replacement therapy as well as high doses of hydrocortisone. ${ }^{56}$ Treating her hyperthyroidism is certain to have reduced several of these risk factors.

The treatment of hypothyroidism, primary or secondary, with exogenous thyroxine in

1 Papadopoulos KI, Hornblad Y, Hallengren B. The occurence of polyglandular autoimmune syndrome type III associated with coeliac disease in patients with sarcoidosis. $F$ Intern Med 1994;236:661-3.

2 Weetman AP. Autoimmunity to steroid-producing cells and familial polyendocrine autoimmunity. Bailliere's Clin Endocrinol Metab 1995;9:157-74.

3 Ingbar SH, Woeber KA. Thyroid gland. In: RH Williams, ed, Textbook of endocrinology. WB Saunders Co Ltd, 1981; pp 180.

4 Michnovicz J, Galbraith RA. Effects of exogenous thyroxine on $\mathrm{C}-2$ and $\mathrm{C}-16 \mathrm{a}$ hydroxylations of estradiol in humans. Steroids 1990;55:22-6. patients with unrecognised adrenal insufficiency, may trigger an adrenal crisis. ${ }^{7}$ The use of glucocorticoids in thyroid storm is due to failure of compensatory endogenous secretion. The presentation of hyperthyroidism as a need for increasing doses of adrenal replacement therapy has not previously been described. This case is a reminder that changes in steroid dose requirements should alert physicians to changes in thyroid gland activity. Anticipatory screening for components of polyendocrinopathy may (and should) lead to earlier diagnosis and treatment in such cases.

\section{Final diagnosis}

Hyperthyroidism in a woman with Addison's disease.

Keywords: Addison's disease; thyrotoxicosis; glucocorticoid replacement therapy; hyperthyroidism

5 Riggs BL, Melton LJ. Involutional osteoporosis. N Engl $\mathcal{F}$ Med 1986;314:1676-86.

6 Wark JD. Osteoporosis: pathogenesis, diagnosis, prevention and management. Bailliere's Clin Endocrinol Metab 1993;7: 151-81.

7 Gordon GG, Southern AL. Thyroid hormone effects on steroid hormone metabolism. Bull NY Acad Med 1977;53: 241-59. 